\title{
Lymphangitic Carcinomatosis of Unknown Primary: A Case Report
}

\author{
Shahin MA ${ }^{1}$, Rahman $\mathrm{M}^{2}$, Dewan $\mathrm{G}^{3}$, Saeed $\mathrm{A}^{4}$, Shazzad $\mathrm{MN}^{5}$, Chowdhury $\mathrm{MR}^{6}$
}

\begin{abstract}
Lymphangitic carcinomatosis is an uncommon manifestation of metastasis to lung. Its presentation can mimic several disorders of the lungs. A case is presented here where patient presented with progressive dyspnoea, dry cough, weight loss and fever. The patient was amenorrheic for the last 2 years. Her physical findings were unremarkable initially other than the weight loss and evidence of pulmonary hypertension. Subsequently her dyspnoea rapidly worsened and the patient became bed bound. Investigations revealed neutrophilic leucocytosis, normal $\mathrm{Hb}$ with high ESR. ANA, anti Cetromere and anti Scl 70 was negative. Serum calcium was normal. Liver function test was normal other than raised alkaline phosphatase. Tuberculin test was negative. Chest X-ray revealed increased bilateral symmetrical streaks and reticulation with peripheral and lower distribution which is consistent with pulmonary lymphangitic carcinomatosis. HRCT of Lungs has shown diffuse infiltrations, reticulo-nodular and ground glass opacities, centrilobular emphysema with accentuation of broncho-vascular densities in both lung fields. There were enlarged mediastinal lymph nodes, bilateral pleural effusion. Doppler Echo revealed mild pulmonary hypertension with fair EF. Tumor markers were done. CA125, CA 19.9 and AFP were ten times higher than normal. Fibreoptic nasopharyngoesophagoscopy was normal. Diagnosis of PLC was made on clinical feature, radiological finding and elevated tumor markers. Symptomatic treatment was given during the period of evaluation. Ultimately the patient succumbed to death. [J Shaheed Suhrawardy Med Coll, 2013;5(2):114-116]
\end{abstract}

Keywords: Pulmonary, lymphangitic carcinomatosis, metastasis, PLC

Received: August 2012; Revised: October 2013; Accepted: November 2013

\section{Introduction}

Lung is one of the most common sites for metastasis ${ }^{1}$. They usually appear as nodular lesions in radiologic images. In some patients, metastasis presents with interstitial spread and it is referred to as Pulmonary Lymphangitic Carcinomatosis (PLC). PLC occurs in 6\%$8 \%$ of patients with pulmonary metastases ${ }^{1}$. Patients with PLC often present with breathlessness and a nonproductive cough. The spread of tumour cells to the pulmonary lymphatic system or the adjacent interstitial tissue results in thickening of the broncho-vascular bundles and septa. Although chest radiographs appear normal for $30 \%-50 \%$ of patients with histologically proven disease ${ }^{2}$, PLC has several characteristic changes that can be observed. Transbronchial biopsy is required for histological diagnosis.

\section{Case Presentation}

A 40-yr-old woman presented with progressive exertional breathlessness, decreasing tolerance to daily activities, dry

1. Dr. Md. Abu Shahin, Assistant Professor, Department of Rheumatology, Banghabondhu Sheik Mujib Medical University, Dhaka

2. Dr. Matiur Rahman, Senior House Officer, United Hospital, Dhaka

3. Dr. Gourab Dewan, Junior Consultant, Rangamati General Hospital, Rangamati

4. Dr. Atia Saeed, Resident, Department of Gastroenterology, Banghabondhu Sheik Mujib Medical University, Dhaka

5. Dr. M. Nahiduzzaman Shazzad, Medical Officer, Department of Rheumatology, Banghabondhu Sheik Mujib Medical University, Dhaka

6. Prof. M.R. Chowdhury, Professor, Department of Rheumatology, Banghabondhu Sheik Mujib Medical University, Dhaka

\section{Correspondence}

Dr. Md. Abu Shahin, Assistant Professor, Room no 1709, Block- D, Department of Rheumatology, Banghabondhu Sheik Mujib Medical University, Dhaka, Bangladesh; Email: abushahin13@yahoo.com; Cell no.: +8801711313416 
cough and significant weight loss during last 6 months. The patient had also history of low grade intermittent fever along with night sweats. No family history of bronchial asthma. Since last 2 years the patient was amenorrheic. On examination there was palpable P2 with loud P2 on auscultation and few inspiratory crackles in lung bases. After hospital admission her dyspnoea rapidly worsened and the patient became totally bed bound. Investigations revealed neutrophilic leukocytosis with high ESR. Autoantibodies for connective tissue diseases were negative. Serum Calcium and LFT were normal except raised alkaline phosphatase. D dimer was raised.

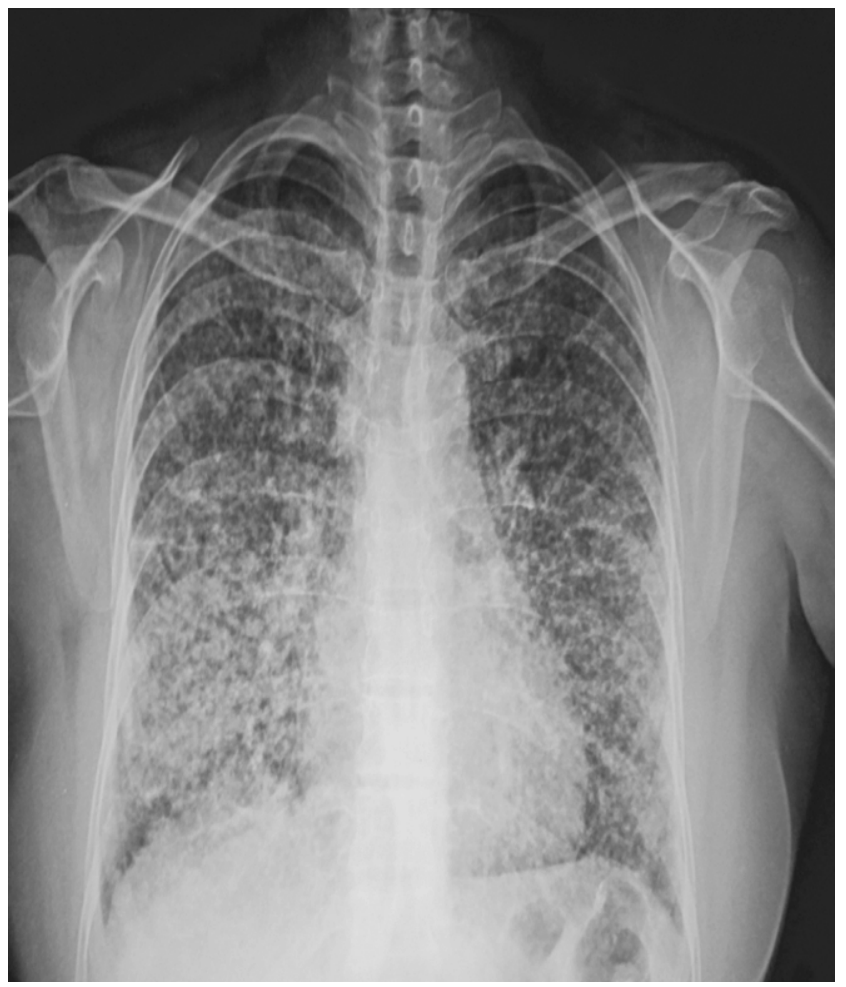

Picture: 1: Bilateral streaks and reticulation predominantly in lower zones.

Chest radiograph (Picture 1) reveals increased bilateral symmetrical streaks and reticulation with peripheral and lower distribution which is consistent with pulmonary lymphangitic carcinomatosis. Chest HRCT (Picture 2) Lungs reveals diffuse infiltrations, reticulo-nodular and ground glass opacities, centrilobular emphysema in both lung fields, accentuation of broncho-vascular densities. There are enlarged mediastinal lymph nodes, bilateral pleural effusion and pleural thickening. Doppler Echo shows mild pulmonary hypertension with fair EF. CA125, CA 19.9 and AFP were ten times higher than normal. Fibre-optic naso-pharyngo-esophagoscopy was normal. Diagnosis of PLC was made on clinical feature, radiological finding and markedly elevated tumor markers. Symptomatic treatment was given during the period of evaluation but ultimately the patient succumbed to death.

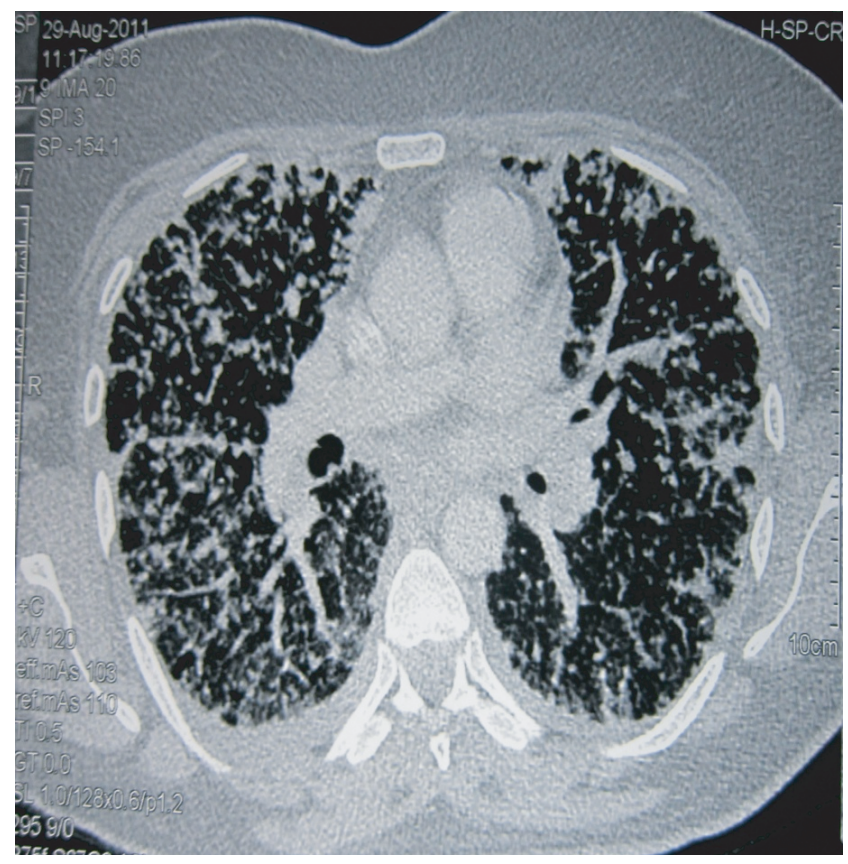

Picture: 2 HRCT scan of chest: Diffuse infiltrations, reticulo-nodular and ground glass opacities, Accentuation of broncho-vascular densities. There are enlarged mediastinal lymph node, bilateral pleural effusion and pleural thickening.

\section{Discussion}

The combination of significant weight loss and worsening exertional breathlessness is common scenario in clinical practice. With this scenario diffuse pulmonary lung disease, infection, malignancy emerges as probable differentials. DPLD, vasculitic disorder, sarcoidosis cannot readily explain the scenario of rapid deterioration. The list of infectious diagnoses is extensive, but the more common disorders include tuberculosis, histoplasmosis, cryptococcosis, brucellosis. The malignancies include metastatic carcinoma, lymphoma, and leukemia.

When considering metastasis, PLC is the most likely form of the disease to present with diffuse infiltrative lung disease. Metastasis to lung usually present as nodular lesions and rarely as PLC. PLC is characterized by diffuse spread of malignancy in the lung, causing inflammation of the lymph vessels. The first reported case of PLC was by Gabriel Andral ${ }^{3}$. Various primary sites have been identified for PLC as discussed before. Eighty percent of them are from adenocarcinomas ${ }^{4-5}$. This patient died before establishment of primary site. While elevated tumor markers suggested underlying malignancy there was no clue in history or clinical examination suggestive of possible site.

It is often difficult to diagnose PLC because symptoms referable to the primary neoplasm are often absent. Recognition of the suggestive history and chest radiographic findings can accomplish an early diagnosis, especially when the patient has a known underlying malignancy. This patient presented with progressive dyspnea and dry cough, fever and weight loss. According to $\mathrm{Babu} \mathrm{et}^{\mathrm{al}}{ }^{6}$ the most common 
symptoms are breathlessness and non productive cough with crackles and without feature of consolidation; however; patients may be relatively asymptomatic. Weight loss is the usual feature of malignancy; our case has marked weight loss than the other reported cases of PLC.

The radiological findings of this patient correlate well with the findings of previous reported cases. Typical radiographic manifestations of PLC include reticular densities and septal lines called Kerley A and B lines. Hilar and mediastinal adenopathy and pleural effusions are frequent additional findings ${ }^{7}$. Notably, the degree of dyspnea may be out of proportion to the radiographic findings in some patients. The differential diagnosis is interstitial lung disease, primary malignancy in the lung, pulmonary sarcoidosis and hypersensitivity pneumonitis.

HRCT is the modality of choice for confirmation of the diagnosis. The findings in CT scan are thickening of interlobular septa, fissures and bronchovascular bundles. These findings may be seen as limited or diffuse and may involve unilateral or bilateral lungs. The radiologic picture may be symmetric or asymmetric in both lungs. The other findings are nodularity in pleura and ground glass opacity ${ }^{10}$. The possibility of interstitial lung disease is to be considered and ruled out. Prakash et $\mathrm{al}^{11}$ described the use of PET/CT in diagnosing PLC and reported that PET/CT has high specificity in detection of PLC.

Histopathological examinations show interstitial oedema and fibrosis along with malignant cells and are found usually on postmortem biopsy. Since the radiological finding in a patient with malignant disease elsewhere is suggestive, a biopsy of the lung is not mandatory ${ }^{11}$. Before a thoracoscopic lung biopsy could be scheduled, our patient's respiratory status worsened, and the patient expired.

PLC often presents in the late stages of malignancy and it indicates poor prognosis ${ }^{12}$. The treatment option is chemotherapy. Cisplatin have been found to be effective ${ }^{13}$. Less than half of patients with PLC who present with respiratory symptoms survive for 3 months, some study reported survival may extends up to 6 months in less aggressive disease with supportive care. Occasionally long term survival is encountered ${ }^{14}$. This patient survived about six and half month since onset of symptoms. Written informed consent was obtained from the patient family for publication of this case report and accompanying images.

\section{Conclusion}

Absence of symptoms or signs attributable to primary source and unusual respiratory symptoms delays diagnosis of PLC. Despite establishment of diagnosis with cellular aetiology even primary source, the condition is fatal. Marked weight loss, rapidly worsening of exertional dyspnoea, dry cough as well as in the background imaging of diffuse reticulonodular lung lesion are the clues of for the suspicion of lymphangitic carcinomatosis. This rare condition need to be considered in appropriate clinical settings.

\section{References}

1. Bruce DM, Heys SD, Eremin O. Lymphangitis carcinomatosa: a literature review. J R Coll Surg Edinb 1996;41(1):7-13

2. Zieske LA, Myers EN, Brown BM. Pulmonary lymphangitic carcinomatosis from hypopharyngeal adenosquamous carcinoma. Head Neck Surg 1988;10(3):195-8

3. Doyle L: Gabriel Andral (1797-1876) and the first reports of lymphangitis carcinomatosa. J R Soc Med 1989;82(8):491-3

4. Yamagishi Y, Akiba Y, Izumiya M, Higuchi H, Iizuka H, Takaishi $H$, Nagata H, Hibi T. A case of advanced gastric cancer with lymphangitis carcinomatosa after operation of Krukenberg tumor treated by TS-1 plus CPT-11 as third-line chemotherapy. Gan To Kagaku Ryoho 2005;32(8):1167-70.

5. Gupta PR, Joshi N, Meena RC, Ali M. Asymptomatic lymphangitis carcinomatosis due to squamous cell lung carcinoma. Indian J Chest Dis Allied Sci 2005;47(2):121-3

6. Babu S, Satheeshan B, Geetha M, Salih S. A rare presentation of pulmonary lymphangitic carcinomatosis in cancer of lip: case report. World Journal Of Surgical Oncology, 2011;9(1): 77; doi:10.1186/1477-7819-9-77

7. Thomas A, Lenox R. Pulmonary lymphangitic carcinomatosis as a primary manifestation of colon cancer in a young adult. CMAJ 2008;179(4):338-40

8. Kirk JE, Kumaran M. Lymphangitis Carcinomatosa as an Unusual Presentation of Renal Cell Carcinoma: A Case Report. J Med Case Reports 2008;2:19

9. Yang SP, Lin CC. Pulmonary lymphangitic carcinomatosis. Taiwan Yi Xue Hui Za Zhi 1968;67(9):361-74

10. Zhang K, Huang Y. Clinical features and diagnosis of pulmonary lymphangitic carcinomatosis. Ai Zheng 2006;25(9):1127-30

11. Prakash P, Kalra MK, Sharma A, Shepard JA, Digumarthy SR. FDG PET/CT in Assessment of Pulmonary Lymphangitic Carcinomatosis. Am J Roentgenol 2010;194(1):231-6

12. Kikuchi N, Shiozawa T, Ishii Y, Satoh H, Noguchi M, Ohtsuka M. A patient with pulmonary lymphangitic carcinomatosis successfully treated with TS-1 and cisplatin. Intern Med 2007; 46(8):491-4.

13. Sawin SW, Aikins JK, Van Hoeuen KH, et al. Recurrent squamous cell carcinoma of the cervix with pulmonary lymphangitic metastasis. Int J Gynaecol Obstet 1995;48(1):85-90

14. Ikezoe J, Godwin JD, Hunt KJ et-al. Pulmonary lymphangitic carcinomatosis: chronicity of radiographic findings in long-term survivors. AJR Am J Roentgenol. 1995;165 (1): 49-52 